\title{
SPLENIC VOLVULUS WITH LACERATION AFTER TRIVIAL TRAUMA IN A CASE OF WANDERING SPLEEN: A RARE CASE REPORT
}

\author{
Vinayak Nanekar ${ }^{1}$, Anjali Wasadikar², Varsha Rote Kaginalkar³, Shweta Gimhavanekar4 \\ ${ }^{1}$ Assistant Professor, Department of Radiodiagnosis, Government Medical College, Aurangabad. \\ ${ }^{2}$ Associate Professor, Department of Radiodiagnosis, Government Medical College, Aurangabad. \\ ${ }^{3}$ Professor, Department of Radiodiagnosis, Government Medical College, Aurangabad. \\ 43rd Year Junior Resident, Department of Radiodiagnosis, Government Medical College, Aurangabad.
}

\begin{abstract}
BACKGROUND

Volvulus of wandering spleen is a rare clinical occurrence with fewer than 500 cases reported and an incidence of less than $0.2 \%$. We present a case of a 27-year-old man, who complained of a short history of severe abdominal pain with the background of recurrent abdominal pain. Ultrasound revealed wandering spleen with splenic vein thrombosis. An abdominal contrast enhanced computerized tomography scan revealed a torted wandering spleen with splenic vein thrombosis with infarction. This required a splenectomy due to splenic infarction. This report highlights the investigations including USG and CECT necessary for a patient who presents with an ischaemic torted wandering spleen.
\end{abstract}

\section{KEYWORDS}

Wandering Spleen, Splenic Volvulus, Infarction, Laceration.

HOW TO CITE THIS ARTICLE: Nanekar V, Wasadikar A, Kaginalkar VR, et al. Splenic volvulus with laceration after trivial trauma in a case of wandering spleen: a rare case report. J. Evolution Med. Dent. Sci. 2016;5(20):1063-1066, DOI: 10.14260/jemds/2016/246

\section{INTRODUCTION}

A wandering spleen is a rare clinical occurrence with fewer than 500 cases reported and an incidence of less than $0.2 \%{ }^{[1,2]}$ The initial clinical diagnosis may be neglected if this rare entity is not kept in mind. Emergency Ultrasonography (USG) could be a very useful initial tool, which reveals absence of the spleen in the left upper quadrant with ectopic spleen.[3] Diagnosis largely depends on imaging studies with computed tomography being the preferred modality. We present a rare case report of volvulus of wandering spleen with splenic infarction and laceration after trivial trauma.

\section{CASE REPORT}

A 28-year-old male patient came to our hospital with intermittent, low-grade abdominal pain for 14 days which worsened in the last 2 days following trivial trauma. He had recurrent episodes of abdominal pain in past years for which he received some medications. On examination he was afebrile, his vitals were stable. There was palpable tender lump in umbilical, right iliac fossa and hypogastric regions. Guarding was present in same regions without rigidity.

Laboratory tests were normal except mild anaemia $(\mathrm{Hb}-$ $9.5 \mathrm{~g} / \mathrm{dL} \%$ ). He was resuscitated with intravenous fluids, given analgesia and referred to ultrasound which revealed absence of spleen in left hypochondrium. Instead, an enlarged spleen (Span $20 \mathrm{~cm}$ ) was present in umbilical, right iliac fossa and hypogastric region with its hilum facing left laterally. It showed altered echotexture. Doppler assessment of the splenic hilum showed normal splenic arterial flow (Fig. 1a),

Financial or Other, Competing Interest: None.

Submission 27-01-2016, Peer Review 26-02-2016,

Acceptance 01-03-2016, Published 10-03-2016.

Corresponding Author:

Dr. Vinayak Nanekar,

Department of Radiodiagnosis,

Government Medical College,

Aurangabad.

E-mail:vnanekar2005@gmail.com

DOI: $10.14260 /$ jemds/2016/246 but no flow was seen in the splenic vein even on power Doppler (Fig. 1b). There was moderate haemoperitoneum. The sonographic findings were concerning for wandering spleen complicated by splenic torsion with likely laceration. Patient was then referred to contrast enhanced CT of abdomen and pelvis.

Enlarged wandering spleen was noted in umbilical, right iliac fossa and hypogastric region without parenchymal enhancement suggesting infarction [Fig. 2 (a \& b), Fig. 4]. The splenic vein was dilated and hyperattenuating on non-contrast CT and showed no enhancement on post-contrast imaging representing thrombosis [Fig. 2 (a \& b)]. Splenic artery was normal. There was twisting of vascular pedicle three times, giving typical whirlpool sign [Fig. 3 (a \& b)]. Splenic hilum was oriented to left lateral side. He also had moderate haemoperitoneum. Rest of the abdominal organs were normal. Diagnosis of wandering spleen with splenic volvulus with splenic vein thrombosis with infarction with haemoperitoneum was given.

Patient underwent open laparotomy. It revealed wandering enlarged spleen in umbilical, RIF and hypogastric region with absence of all splenic ligamentous attachments. Splenic vessels were torted with about 1080 degrees clockwise rotation with long vascular pedicle with splenic vein thrombosis (Fig. 5). There was splenic laceration of size $4 \times 3 \times 3$ $\mathrm{cm}$ at mid pole (Fig. 6) with moderate haemoperitoneum. As spleen was completely infarcted, total splenectomy was performed. The patient's post-operative course was uneventful. The patient was discharged with appropriate postsplenectomy treatment.

\section{DISCUSSION}

The spleen begins its development from a mass of mesenchymal cells in the dorsal mesogastrium in the fifth week of gestation. A counter clockwise rotation of the foregut and elongation of the mesogastrium allow it to assume its normal anatomical position in the left upper quadrant. During this migration, the spleen establishes its peritoneal connections with the left kidney and stomach through 
splenorenal and gastrosplenic ligaments. When these ligaments are congenitally absent or abnormally elongated, it is described as wandering spleen. ${ }^{[4]}$

A wandering spleen can be acquired form, in which hormonal effects of pregnancy and abdominal wall laxity are proposed as determining factors.[5-6]

A wandering spleen usually presents between the ages of 20 and 40 years, being more common in women. Children make up one-third of cases with an equal preponderance in boys and girls under 10 years.[7] Our patient was 28 years old male.

In patients with wandering spleen, torsion can be intermittent causing symptoms of mild abdominal pain related to splenic congestion or it can be acute and severe, leading to rapid splenic necrosis. Complications include pancreatitis, bowel obstruction, gastric volvulus, gastric and duodenal compression and most commonly splenic infarction.[8] Our patient presented with acute pain in abdomen in background of recurrent abdominal pain.

Laboratory tests are usually non-specific. While physical exam often reveals a painful abdominal mass, the diagnosis is generally made by cross-sectional imaging. ${ }^{[9-11]}$

Ultrasound often demonstrates an enlarged, ectopic spleen. Splenic echotexture varies by the degree of parenchymal congestion and/or infarction. $[9,10,12]$

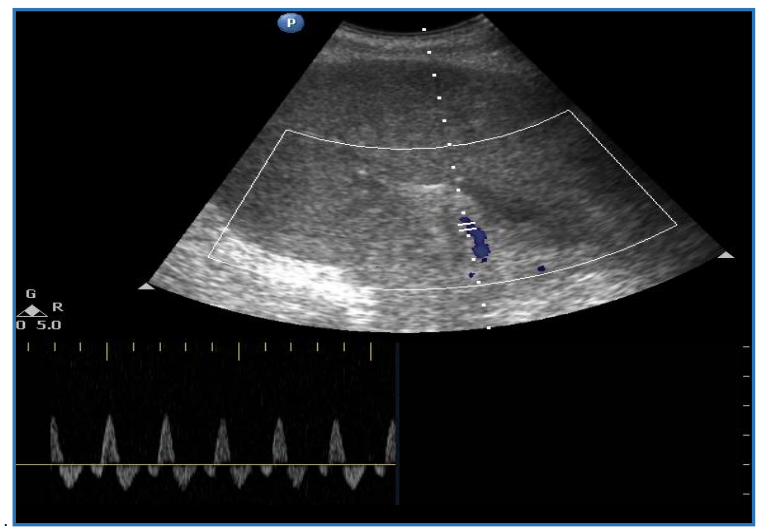

The drawbacks of ultrasound include limitation by bowel gas, difficulty in demonstrating the twisted pedicle, the varied appearances of splenic infarction and inability to accurately assess status of adjacent viscera.

Similar findings can be seen with contrast enhanced CT including an enlarged ectopic spleen with relative hypoattenuation, suggesting poor perfusion.[9,10,12] In some cases, twisting of the vascular pedicle may demonstrate a "whorled" appearance.[9] CT also demonstrates secondary findings like haemoperitoneum and entrapment of adjoining viscera secondary to torsion.

In our case, diagnosis of splenic torsion with infarction was made on USG and colour Doppler and later confirmed on CECT. Also, it gave detailed preoperative evaluation of degree of torsion.

Splenic torsion is treated surgically with detorsing of the vascular pedicle and splenopexy if the spleen is found to be viable or with splenectomy if the spleen is already necrotic.

Preservation of the spleen by different surgical techniques has been the focus of modern therapeutic concepts for the treatment of ectopic spleen.[11,13] Presently, splenic surgery by laparoscopic approach is preferred technique because it is less painful and overall less morbidity. In present case, splenectomy was done because of infarction.

Fig. 1(a) \& (b): Sagittal scan of abdomen reveals normal splenic artery showing triphasic flow (Left) \& splenic vein thrombosis (Right)

Note enlarged spleen showing heterogeneous echotexture and its hilum facing inferiorly \& to left
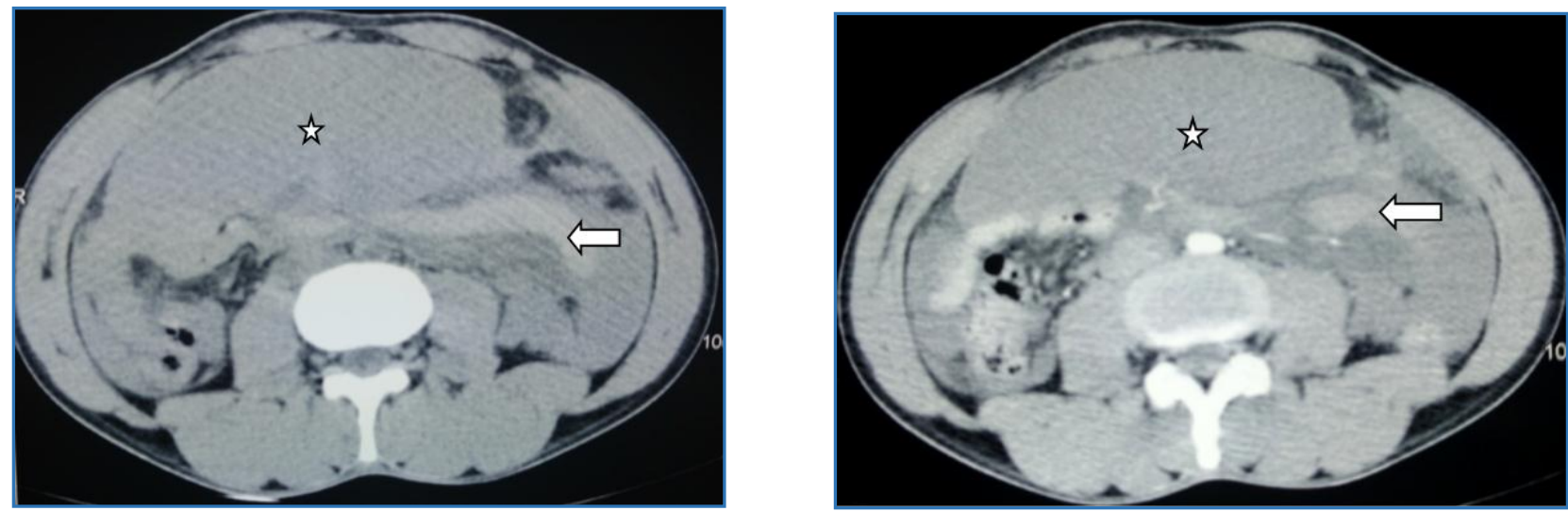

Fig. 2: (a) Pre and (b) Post contrast axial CT images show no definite change in the attenuation of the splenic parenchyma (Star) denoting complete infarction

Note hyperdense dilated non-enhancing splenic vein (Open arrow) suggesting thrombosis 

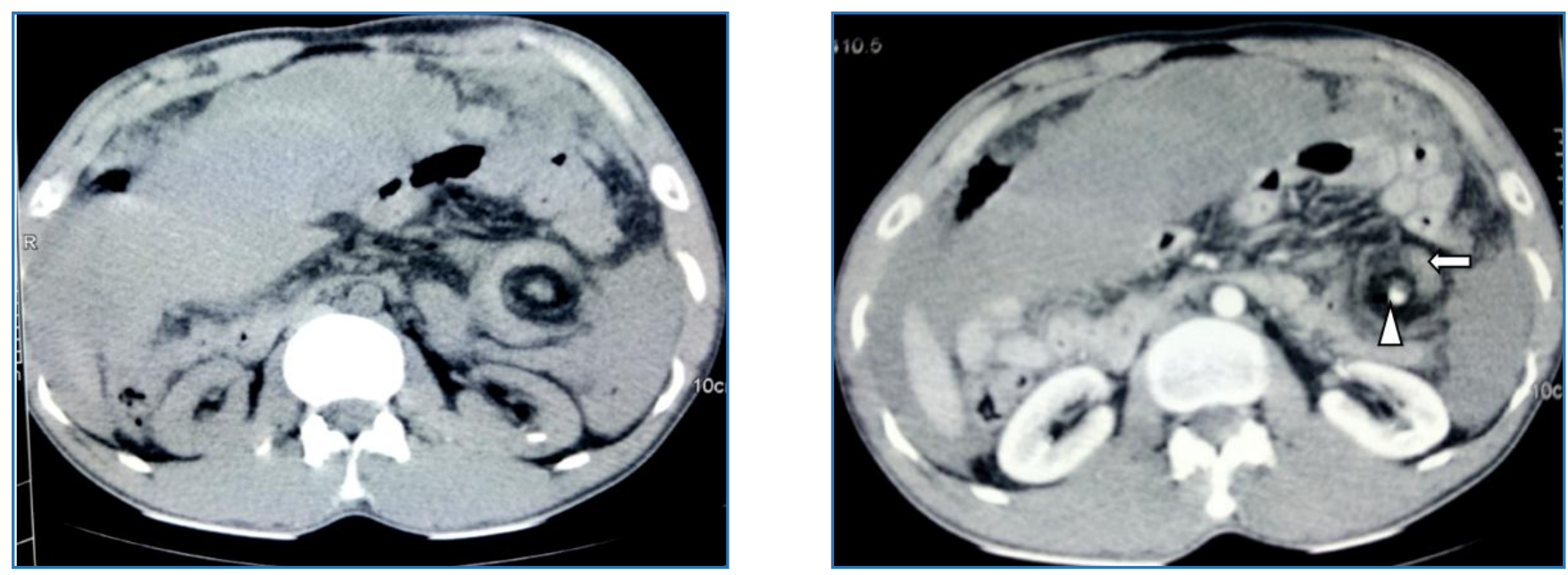

Fig. 3 ( $a$ \& b): (a) Pre and (b) Post contrast axial CT images showing twisting of vascular pedicle at splenic fossa giving "Whirlpool sign"

Note normally enhancing splenic artery (Arrowhead) \& non-enhancing splenic vein (Open arrow)

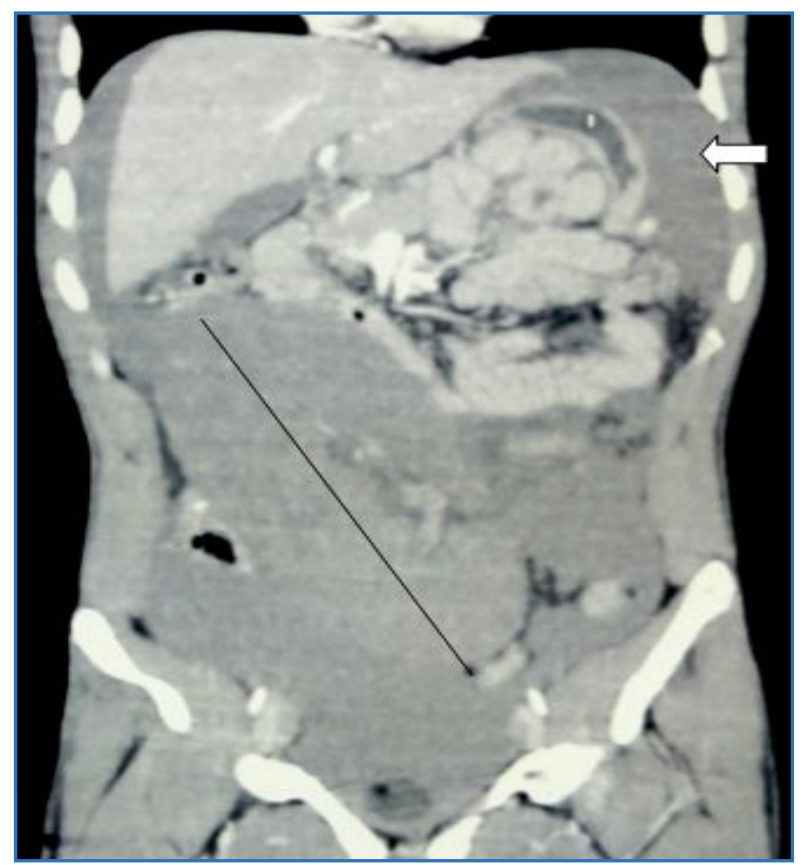

Fig. 4: Coronal reformatted CT showing enlarged ectopic spleen (Black line) with empty splenic fossa containing haemoperitoneum (Open arrow)

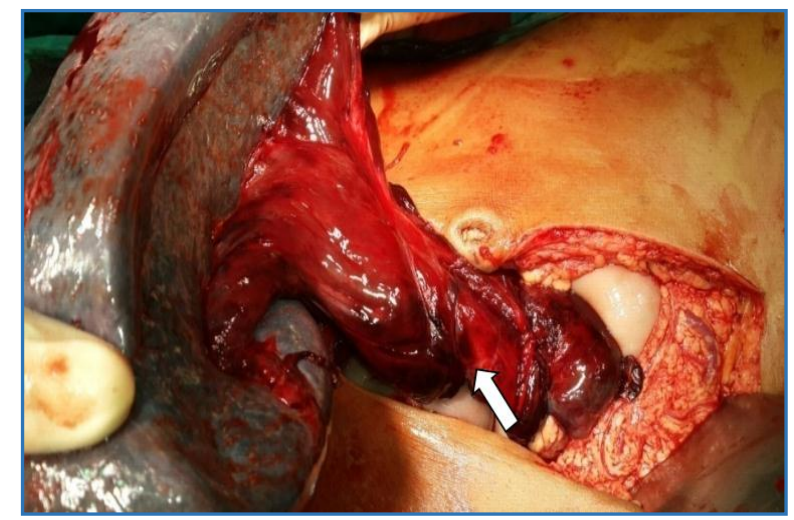

Fig. 5: Intraoperative image showing twisting of vascular pedicle with splenic vein thrombosis (Arrow)

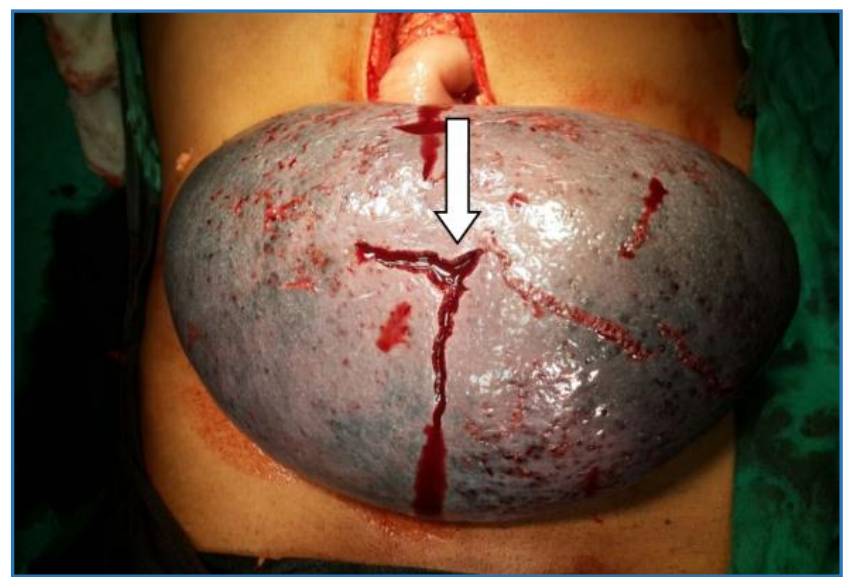

Fig. 6: Intraoperative image showing splenic laceration at midpole (Arrow)

\section{CONCLUSION}

Wandering spleen with or without volvulus is a rare clinical entity. Imaging modalities play an important role in the diagnosis. Absence of spleen in the left upper quadrant with absent flow in splenic vessels on ultrasound should alert the possibility of the diagnosis. Contrast enhanced CT scan confirms the diagnosis and detect any complications. CT is also required for complete preoperative evaluation and in deciding whether splenopexy or splenectomy is required.

It is sometimes difficult to diagnose splenic laceration on CT, particularly when spleen is completely infarcted as in our case. Predisposing factors of laceration in our case may be superficial hugely enlarged spleen. Hence, there should be a high degree of suspicion for splenic laceration when patient with wandering spleen has haemoperitoneum in background of history of trivial trauma.

\section{REFERENCES}

1. Raissaki M, Prassopoulos P, Daskalogiannaki M, et al. Acute abdomen due to torsion of wandering spleen: CT diagnosis. Eur Radiol 1998;8:1409-1412.

2. Satydas T, Nasir N, Bradpiece HA. Wandering spleen: case report and literature review. J R Coll of Surg Edin 2002;47:512-514. 
3. Steinberg R, Karmazyn B, Dlugy E, et al. Clinical presentation of wandering spleen. J Pediatr Surg 2002;37:E30.

4. Moore KL, Persaud TVN. The digestive system. In: Moore KL, Persaud TVN, eds. The developing human, clinically oriented embryology. Philadelphia, PA: WB Saunders Co. 1998;6th ed:271-302.

5. Gomez D, Patel R, Rahman SH, et al. Torsion of a wandering spleen associated with congenital malrotation of the gastrointestinal tract. Int J Radiol 2006;5:1.

6. Ben Elya A, Zissinb R, Copela L, et al. The wandering spleen: CT findings and possible pitfalls in diagnosis. Clin Radiol 2006;61:954-958.

7. Dawson JH, Roberts NG. Management of the wandering spleen. Aust N Z J Surg 1994;64:441-444.
8. Karmazyn B, Steinberg R, Gayer G, et al. Wandering spleen-the challenge of ultrasound diagnosis: report of 7 cases. J Clin Ultrasound 2005;33:9.

9. Swischuk LE, Williams JB, John SD. Torsion of wandering spleen: the whorled appearance of the splenic pedicle on CT. Pediatr Radiol 1993;23:476-7.

10. Brocker FHL, Fellows K, Treves S. Wandering spleen in three children. PediatrRadiol 1978;6:211-4.

11. Soleimani M, Mehrabi A, Kashifi A, et al. Surgical treatment of patients with wandering spleen: report of six cases with a review of the literature. Surgery Today 2007;37:261-9.

12. Chawla SC, Boal DKB, Dillon PW, et al. Best cases of AFIP: splenic torsion. Radiographics 2003;23:305-8.

13. Cho SA, Choh NA, Dar I, et al. Wandering spleen presenting as recurrent abdominal pain in a young female. Indian J Pediatr 2008;75(11):1182. 